\title{
Good things come in small packages: New trends in acquisition of remotely-sensed data
}

\author{
Robert Bergquist, ${ }^{1}$ Sherif Amer ${ }^{2}$ \\ ${ }^{1}$ Ingerod, Brastad, Sweden; ${ }^{2}$ Department of Urban and Regional Planning and Geo-Information \\ Management, Faculty of Geo-Information Science and Earth Observation, University of Twente, \\ The Netherlands
}

As of 1 January, 2019, no less than 4,987 orbiting satellites were traced by the United Nations Office for Outer Space Affairs. While, most of the early launches lifted several tons into space with some payloads big enough to provide living space for several persons, i.e. the International Space Station, a new trend has taken hold. Apart from being put into orbit by private companies, such as Maxar Technologies, OneWeb, Planet Labs, SpaceX, etc. rather than national space agencies, many of the latest of satellites typically weigh less than five $\mathrm{kg}$, allowing them to be put into orbit with minimal expenditure. With 150 such satellites in operation as of September 2018, Planet Labs has currently the largest fleet of any company, while OneWeb is busy launching an initial network of 648 mini-satellites planned to provide worldwide Internet access by 2021. In order to reach every conceivable spot on Earth, another 300 such satellites will be added to this network in the next few years.

Although the space around Earth already seems to be burdened with an overload of satellites, it is predicted that the number would double as early as in 2027 . The various orbits are, however, divided by altitude into separate bands. At the top we find the geostationary weather satellites operated by the U. S. National Oceanic and Atmospheric Administration (NOAA). In order to remain over the same area on the ground, their altitude must be very close to $35,786 \mathrm{~km}$, high enough to cover half the globe in the optical scenes transmitted to the receiving stations. The next rung, 705-850 km above the ground, comprises relatively wellknown satellites with applications in meteorology, climatology and oceanography, such as the Landsats with their 15/30-m optical resolution launched by the NASA as well as NOAA's polar-orbiting satellites with their 1-km resolution Advanced Very High Resolution Radiometer. These satellites collect detailed environmental information on land cover, meteorological conditions, var-

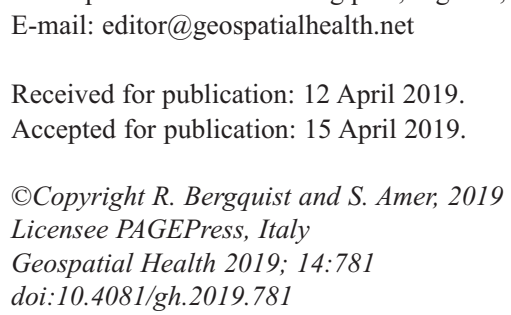

This article is distributed under the terms of the Creative Commons Attribution Noncommercial License (CC BY-NC 4.0) which permits any noncommercial use, distribution, and reproduction in any medium, provided the original author(s) and source are credited. ious environmental variables and air pollutants. In addition, the Japanese Aerospace Exploration Agency provides a Public-health Monitoring and Analysis Platform delivering data on rainfall, soil moisture, aerosol optical thickness, land surface temperature, elevations as well as various indices, e.g., the NDVI. This system is designed to enable users to download and utilize data without the need for additional data processing (Oyoshi et al., 2019). Sensors onboard all these satellites play important roles for epidemiological analyses when in-situ data are unavailable or spatially and/or temporally sparse.

Still lower, between 480 and $700 \mathrm{~km}$ above the surface, we find the traditional, relatively voluminous, high-resolution satellites specializing in sub-meter imagery, which all have orbital periods as short as 1.5 to 2 hours because of the comparatively high speed required at that level to avoid falling back to Earth. This band of satellites includes GeoEye, SPOT, Pléiades and WorldView (for details see Bergquist and Manda, 2019) and at the 400-km altitude, Planet Lab's 150 mini-satellites. Although the spatial resolution of the latter cannot rival WorldView's $31 \mathrm{~cm}$, their higher speed due to their lower altitude (400 compared to 600 $\mathrm{km}$ ) and their plurality help to keep revisit times very short. Planet Lab is also moving from just transmitting observed data to also indicate perceived risk explored through machine-learning applied to its imagery feeds. This is a novelty which not only improves detection but also prioritizes resources improving the speed at which the agency extracts vital information allowing their clients to spot trends early and be alerted to changes without delay.

The remote-sensing landscape is also changing with the advent of the commercially available, unmanned aerial vehicles (UAVs). Generally called drones and first thought of only as children's playthings, they have developed into various serious applications. Although UAVs cannot replace satellite-generated remote-sensing with respect to collection of environmental data, they should be seriously contemplated with respect to user-set agendas. Bridging the $400-\mathrm{km}$ gap between the lowest flying satellites and the ground, UAVs can be sent into ecological niches for close-up photography and sample collection in areas that are difficult to reach or which should preferably not be disturbed. The use of small UAVs has already attracted considerable scientific interest in the field of public health other than data collection. A short scan of the literature shows quite a variety of potential and real UAV use in the public health arena such as: i) telemedicine, i.e. the establishment of wireless communication between local health staff and specialists (Rosser et al., 2018); ii) responding to health care demand of medical services, such as delivery of medications and medical devices to patients in difficult-to-reach areas (McCall, 2019); iii) transporting medications and blood samples between remote clinics and hospitals; iv) responding to emergency calls assessing injuries, directing rescue and relief opera- 
tions (Estrada and Ndoma, 2019); v) aid delivery to refugees and victims of war; vi) collection of some high-resolution, spatio-temporal data at a relatively low cost; and vii) high-accuracy detection and precision-targeting of spatial interventions, e.g. mapping malaria vector breeding sites (Hardy et al., 2017) or delivering baits to animals that act as disease carriers (Yu et al., 2017).

A recent example of the use of UAVs in the veterinary field is the assessment of the population dynamics of Galba truncatula, the intermediate host snail for Fasciola hepatica by characterization of suitable small water bodies (Charlier et al., 2014). They found that while rainfall and soil type information is easily accessible through satellite-generated data, drones lend themselves to close examinations allowing a better delineation of these water bodies.

Another possible approach would be to use UAVs to target animal hosts for parasites such as Echinococcus and Leishmania or carriers of viral infections such as rabies. The former has been reported from China where wild dogs, the common definitive hosts for the parasites causing alveolar and cystic echinococcosis, were dewormed by leaving baits in nature containing praziquantel. Savings up to $67 \%$ of the overall cost were shown by UAV bait delivery compared to manual delivery (Yu et al., 2017), while an unsuccessful manual vaccine bait distribution campaign against rabies in wild foxes in Italy, was exchanged for bait distribution by helicopters using a satellite-navigated, computer-supported, automatic bait drop system (Mulatti et al., 2011). Emulating the Chinese approach, UAV could probably have been used also in this case.

Overall, UAVs evade many of the limitations of satellite-generated data, such as long repeat times, cloud cover, the high cost of satellite-generated, high-resolution, up-to-date imagery and other products. In addition, they operate close to the ground allowing close-ups of areas difficult to reach in other ways. The disadvantages are that only comparatively limited geographic areas can be served, flights can be limited by environmental situations such as high wind speeds and rainfall, sometimes also by extreme humidity and temperature, and the available sensors are not of the same high quality as those onboard satellites (but rapidly improving). From the point of view of legal aspects, there is also the question of invasion of privacy; potential collisions with aircraft, distur- bance of wildlife and association with warfare that needs to be taken into account.

\section{References}

Bergquist R, Manda S, 2019. The world in your hands: geohealth then and now. Geospat Health 14:3-16.

Charlier J, Soenen K, De Roeck E, Hantson W, Ducheyne E, Van Coillie F, De Wulf R, Hendrickx G, Vercruysse J, 2014. Longitudinal study on the temporal and micro-spatial distribution of Galba truncatula in four farms in Belgium as a base for small-scale risk mapping of Fasciola hepatica. Parasit Vectors 7:528.

Estrada MAR, Ndoma A, 2019. The uses of unmanned aerial vehicles - UAV's - (or drones) in social logistic: natural disasters response and humanitarian relief aid. Procedia Comput Sci 149:375-83.

Hardy A, Makame M, Cross D, Majambere S, Msellem M, 2017. Using low-cost drones to map malaria vector habitats. Parasit Vectors 10:29.

McCall B, 2019. Sub-Saharan Africa leads the way in medical drones. Lancet 393:17-8.

Mulatti P, Ferrè N, Patregnani T, Bonfanti L, Marangon S, 2011. Geographical information systems in the management of the 2009-2010 emergency oral anti-rabies vaccination of foxes in north-eastern Italy. Geospat Health 5:217-26.

Oyoshi K, Mizukami Y, Kakuda R, Kobayashi Y, Kai H, Tadono T, 2019. Japan Aerospace Exploration Agency's Public-health Monitoring and Analysis Platform: a satellite-derived environmental information system supporting epidemiological study. Geospat Health 14:103-10.

Rosser JC Jr., Vignesh V, Terwilliger BA, Parker BC, 2018. Surgical and medical applications of drones: a comprehensive review. JSLS 22:e2018.00018.

Yu Q, Xiao N, Yang SJ, Han S, 2017. Deworming of stray dogs and wild canines with praziquantel-laced baits delivered by an unmanned aerial vehicle in areas highly endemic for echinococcosis in China. Infect Dis Poverty 6:117. 